\title{
$-58-$ \\ EXPLORATION EN CONTINU DE LA FONCTION RESPIRATOIRE D'UN POISSON : GARDONUS RUTILUS
}

\author{
par J. GORIN (1), I. LIBERMANN (2) et P. HARICHAUX (3)
}

\begin{abstract}
RESUME :
L'étude complète des échanges gazeux respiratoires d'un poisson avec son milieu ambiant semble nécessiter, à première vue, d'une part des installations importantes et d'autre part l'analyse ponctuelle de prélèvements d'eau.

La méthode personnelle présentée a l'avantage de transcrire en continu les variations de pressions partielles d'un milieu hydrique où vit un poisson, ce qui traduit indirectement mais avec une sensibilité suffisante toutes les modifications naturelles ou provoquées de l'activité respiratoire du poisson dans ce milieu avec lequel il échange.
\end{abstract}

Simple à mettre en œuvre au laboratoire, elle est susceptible d'applications dans des domaines aussi variés que l'étude du comportement, l'éco-physiologie de la respiration, la toxico-physiologie ou la pathologie.

\section{INTRODUCTION}

En pratique, on a l'habitude d'exprimer le taux d'un gaz dissous dans l'eau en $\mathrm{mg} /$ litre, comme on le fait pour la concentration des différents ions. Or, un liquide, dans les conditions naturelles, est surmonté d'une phase gazeuse, l'air ambiant, qui exerce par unité de surface une force appelée pression atmosphérique. Cette pression tendra à dissoudre l'air dans le liquide : il s'instaure ainsi un équilibre de pressions gazeuses entre le compartiment aérien d'une part et le compartiment aquatique d'autre part.

Par ailleurs, l'air est composé de plusieurs gaz dont chacun exerce sa propre pression, dite pression partielle (la somme des pressions partielles étant évidemment égale à la pression atmosphérique).

Par application de ces deux principes, l'air sec contenant $20,9 \%$ d'oxygène, sous la pression atmosphérique normale de $760 \mathrm{~mm} \mathrm{Hg}$ (ou 760 torr), la pression partielle de l'oxygène $\left(\mathrm{PO}_{2}\right)$ dans l'air vaudra $760 \times 20,9 \%$, soit approximativement $160 \mathrm{~mm} \mathrm{Hg}$. Si on introduit de l'air dans l'eau, à l'équilibre des pressions gazeuses, ou équilibre tonométrique, la pression partielle $\mathrm{PO}_{2}$ de l'eau sera égale à la $\mathrm{PO}_{2}$ atmosphérique.

(1) Laboratoire de Physiologie, Professeur P. HARICHAUX, Faculté de Médecine, 12 rue Frédéric Petit, 80036 AMIENS Cedex.

(2) Departamento de Patologia y Fisio-patologia, Facultad de Medicina, MONTEVIDEO (URUGUAY).

(3) Expert Régional en Hydrobiologie, nommé par arrété préfectoral. 
Cette notion prend toute son importance en biologie où c'est, en définitive, par le jeu des différences de pressions partielles que s'effectuent les transferts gazeux, avec le milieu ambiant (oxygène, gaz carbonique, azote ou autre) de tous les organismes vivants, aussi bien dans les conditions de la respiration aérienne que de la respiration aquatique.

Dans cette optique, il nous a paru justifié d'étudier le comportement respiratoire d'un poisson vis-à-vis de son milieu par une méthode nouvelle utilisant des èlectrodes spécifiques aux pressions partielles d'oxygène et de gaz carbonique (HARICHAUX et coll., 1971, 1974). Nous nous sommes plus particulièrement intéressés aux variations de pressions partielles gazeuses du milieu dans les conditions du confinement, en excluant toutes mesures au niveau du sang.

Dans ces conditions, la $\mathrm{PO}_{2}$ du milieu représente une résultante à la fois de respiration de l'animal et des conditions extérieures pouvant elles mêmes modifier la $\mathrm{PO}_{2}$.

Notre dispositif pourrait préfigurer une * représentation sectorielle • du bilan d' $\mathrm{O}_{2}$, notamment décrite par ARRIGNON (1973), ou, comme nous y travaiłions actuellement, à titre d'application, une * détermination rapide du comportement des rivières face à l'accroissement potentiel de pollution représenté par de nouvelles implantations industrielles *. (*)

\section{MATERIEL ET METHODE (fig. 1)}

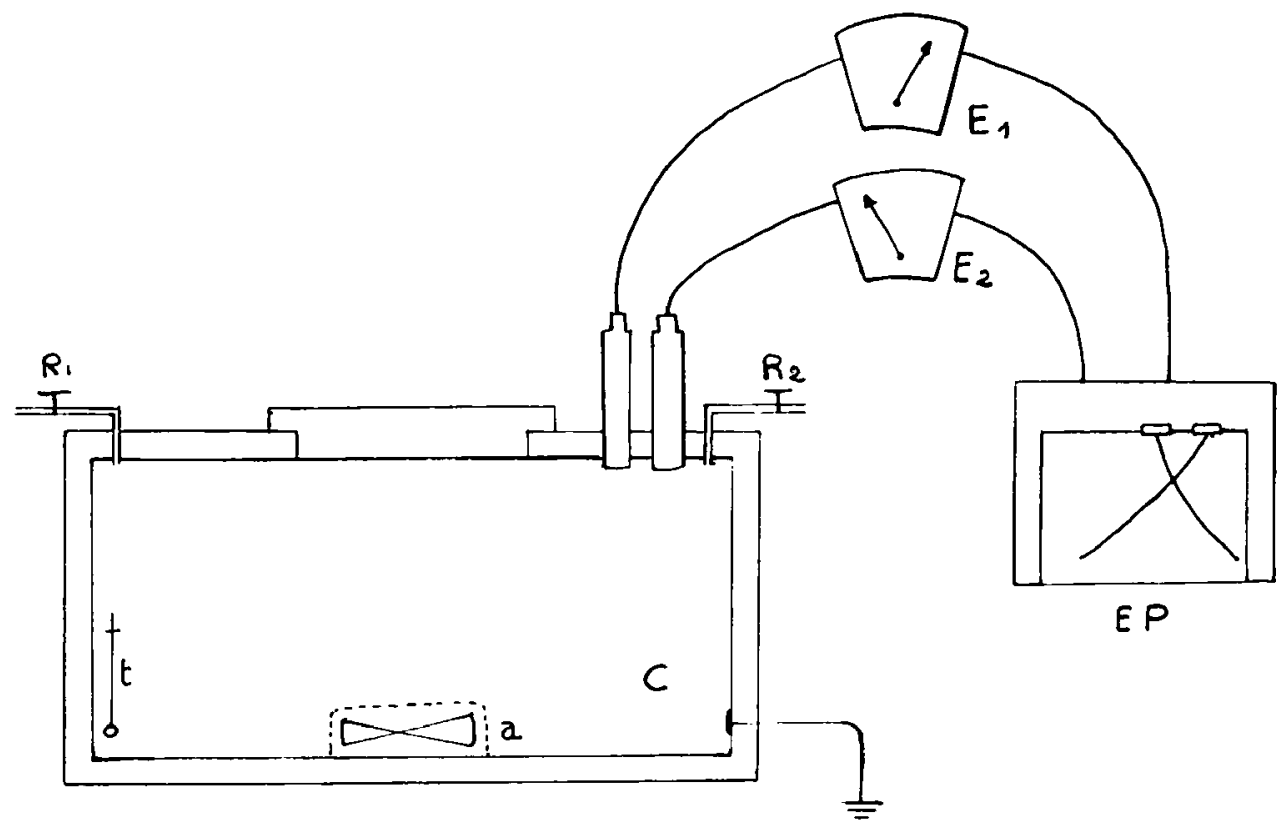

Fig. 1 : Schéma du dispositif expérimental

Une chambre en plexiglass (C) maintenue à temperature constante ( $t$ ) contient le poisson à étudier. Une agitation modérée (a) permet l'homogénéisation du milieu. Deux électrodes à $\mathrm{PO}_{2}$ et $\mathrm{PCO}_{2}$ desservent chacune un électromètre $\left(E_{1}\right),\left(E_{2}\right)$ et un enregistreur potentiométrique bipiste (EP). Deux robinets $R 1$ et R2 permettent l'adjonction de substances dans le milieu.

(*) Travail sous subvention de la Préfecture de Région de Picardie. 
21. Notre dispositif expérimental comporte une enceinte en plexiglass complètement fermée, contenant une eau de composition électrolytique connue $\left.{ }^{*}\right)$, préalablement tonométrée sur l'air et dans laquelle on a introduit un poisson: Gardonus rutilus $\left({ }^{* *}\right)$.

22. Une chaine de mesures comprend deux électrodes à $\mathrm{PO}_{2}$ et $\mathrm{PCO}_{2}$ qui desservent chacune un électromètre et un enregistreur potentiométrique bipiste, ou à deux entrées $x y$, qui transcrit en continu les variations de pressions partielles d'oxygène et de gaz carbonique du milieu hydrique.

23. Le dispositif est placé dans une chambre thermostatée à $10 \circ \mathrm{C}$ augmentant ainsi la stabilité des mesures et des conditions métaboliques des sujets.

\section{RESULTATS}

31. Analyse des enregistrements en continu de $\mathrm{PO}_{2}$ et $\mathbf{P C O}_{2}$ en fonction des temps :

311. La courbe $\mathrm{PO}_{2}=f(t)$ peut être décomposée généralement en 3 parties: la première montre une chute souvent brutale de la $\mathrm{PO}_{2}$, de durée plus ou moins longue traduisant l'adaptation du poisson aux conditions expérimentales; la pente diminue alors et se régularise en devenant approximativement linéaire jusqu'à 10 à 5 torr, où elle tend de manière asymptotique vers zéro. Durant cette dernière phase, dont la durée est d'environ deux heures, l'équilibration de l'animal est perturbée, mais si la réoxygénation du milieu intervient juste avant l'arrêt des mouvements respiratoires, l'animal récupère son équilibration normale.

Gardonus rutilus semble supporter, sans dommage apparent, l'hypoxie sévère du milieu, même après des expériences répétées évoquant l'existence de processus aniérobies.

312. Corrélativement à la décroissance de la $\mathrm{PO}_{2}$, les enregistrements montrent une augmentation de la $\mathrm{PCO}_{2}$ du milieu. A l'inverse de la $\mathrm{PO}_{2}$, la $\mathrm{PCO}_{3}$ ne tend pas vers une limite, ce qui nous a conduits à enregistrer directement la $\mathrm{PCO}_{2}$ en fonction de la $\mathrm{PO}_{2}$ (fig.2).

(*) Référence $111 / 1$ 128/72-F de la direction générale des Aftaires Industrielles Tachnologiques et Scientifiques, à la Commission des Communautés Européennes.

$\left({ }^{*}\right)$ Nous remercions tout particulierement $M$. DEBRUILLE, Garde-Chef du CSP, d'avoir bien voulu nous fournir gracieusement tous les animaux nézessaires. 


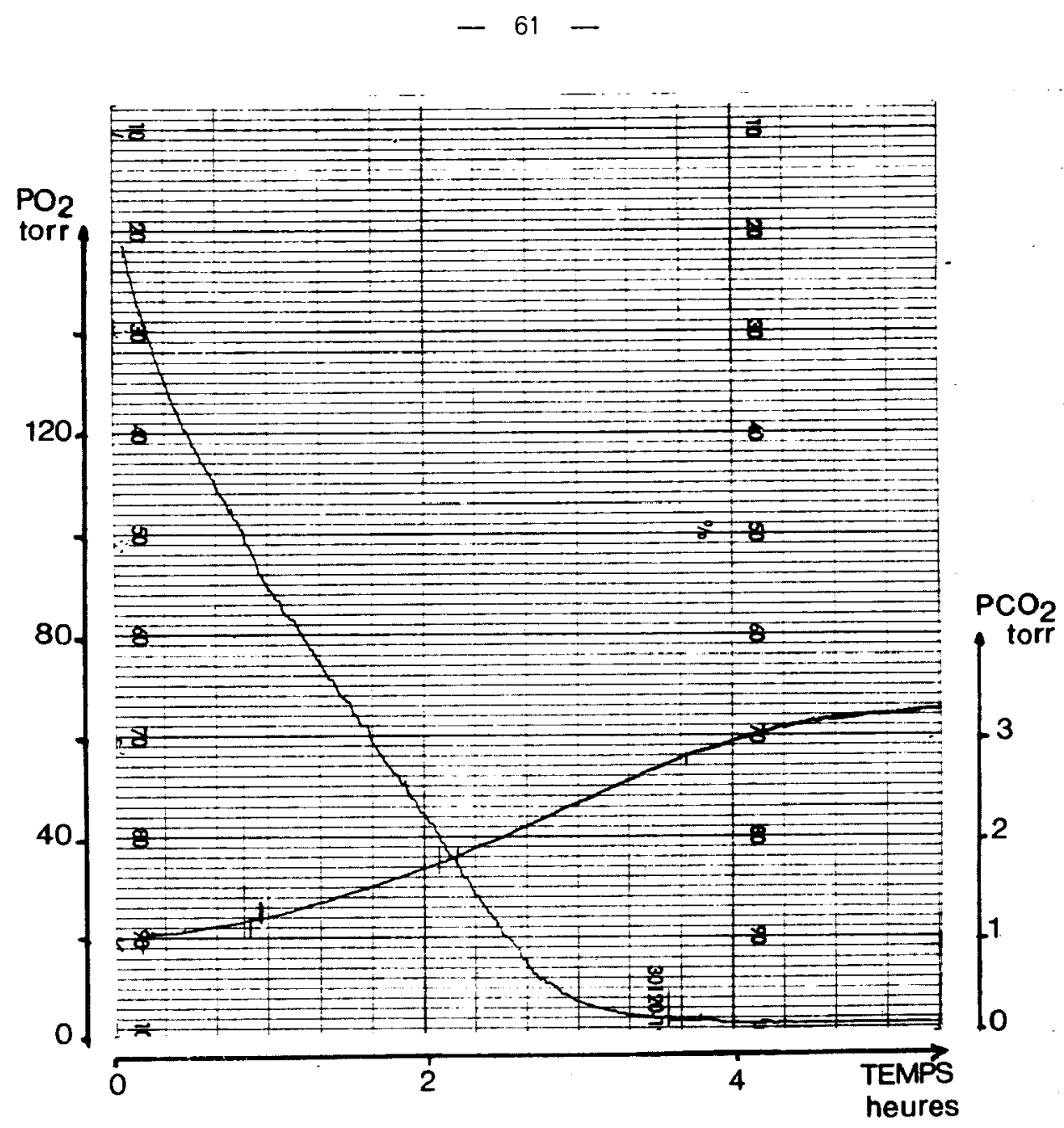

Fig. 2: Evolution de la $\mathrm{PO}_{2}$ et $\mathrm{PCO}_{2}$ en fonction du temps

313. Calcul de la consommation d'oxygene $\left(\mathrm{VO}_{2}\right)$ :

A partir des enregistrements, il est possible de calculer la $\mathrm{VO}_{2}$ moyenne et instantanée à partir de la relation dérivée de la Loi de Henry.

$$
\dot{V}_{2}=\frac{x \mathrm{O}_{2} V \perp \mathrm{PO}_{2}}{760 \mathrm{Jt}} \text { où }
$$

$x \mathrm{O}_{2}$ : est le coefficient de solubilite de l'oxygene dans l'eau, exprimé en $\mathrm{ml}$ de gaz par $\mathrm{ml}$ de liquide, à la température considérée et à $760 \mathrm{~mm} \mathrm{Hg}$.

$\checkmark$ : le volume de l'enceinte.

760 : la pression atmosphérique normale.

\lrcorner $\mathrm{PO}_{2}$ : la variation de $\mathrm{PO}_{2}$ sur un intervalle de temps $\perp t$. 
Si la formule permet en outre le calcul de la $\overline{\mathrm{V}} \mathrm{CO}_{2}$, les résultats doivent cependant être interprétés en raison de l'éventuel pouvoir tampon de l'eau qui influe sur l'équilibre carbonique du milieu (DEJOURS et coll., 1968), et de la forme chimique du $\mathrm{CO}_{2}$ rejeté.

\section{Evolution de la $\mathrm{PCO}_{2}$ en fonction de la $\mathrm{PO}_{2}$ :}

Un enregistreur potentiométrique en $x y$ nous a permis de suivre en continu le diagramme de Fenn et Rahn $\mathrm{PCO}_{2}=f\left(\mathrm{PO}_{2}\right)$ et de constater d'importantes variations du quotient respiratoire $(R)$ selon les individus. En outre la pente n'est pas linéaire entre le début et la fin de l'expérience: on constate une élévation brusque de la $\mathrm{PCO}_{2}$ dans les basses valeurs de la $\mathrm{PO}_{2}$, bien plus importante quand plusieurs poissons sont utilisés ensemble, et, dans ce cas, la hauteur du pic est fonction de la $\mathrm{PO}_{2}$ initiale (HARICHAUX et Coll., 1973) (fig. 3).

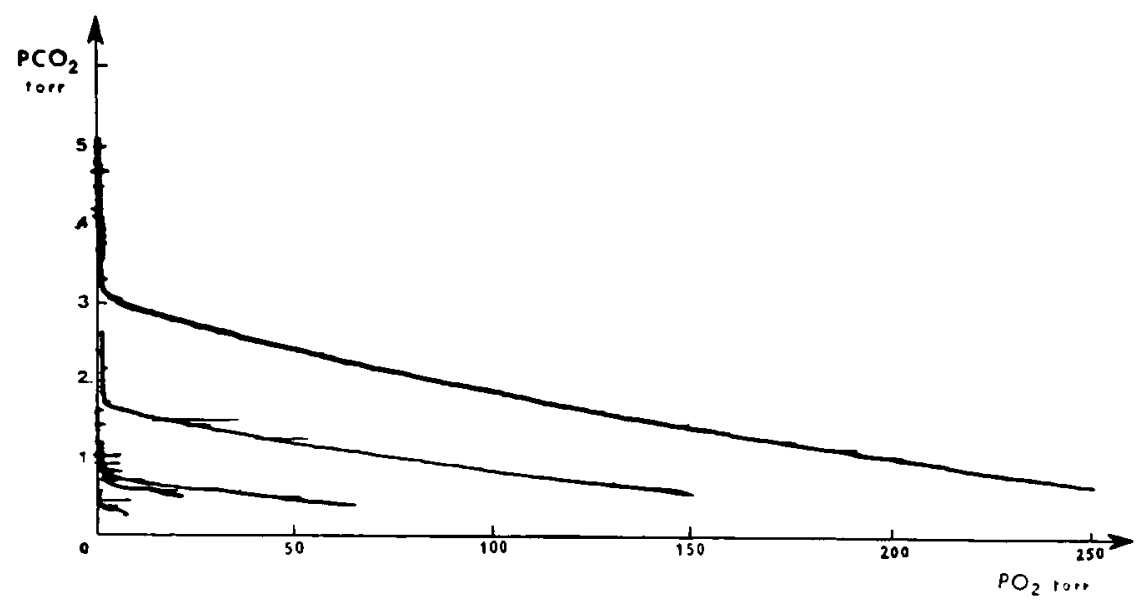

Fig. 3: Enregistrements directs de $\mathrm{PCO}_{2}$ en fonction de $\mathrm{PO}_{2}$ de trois Gardons réunis dans l'aquarium, et pour différentes valeurs de la $\mathrm{PO}_{2}$ initiale 
La confrontation de nombreux résultats laisse supposer une double possibilité de rejet du $\mathrm{CO}_{2}$ dans le milieu: sous forme soluble d'une part, et d'autre part sous forme de bicarbonates qui se dissocient lentement dans le milieu. (Ces résultats sont à rapprocher de ceux de MAREN, 1967).

Nous avons noté par ailleurs une reproductibilité très satisfaisante de tous les tracés, dans ces conditions d'enregistrement direct de $\mathrm{PCO}_{2}=f\left(\mathrm{PO}_{2}\right)$, ce qui n'est pas le cas pour les enregistrements des variations de $\mathrm{PCO}_{2}=f(t)$ ou $\mathrm{PO}_{2}=f(t)$.

33. Variations de la $\mathrm{VO}_{2}$ :

Si le poids et la température influencent de façon notable la $\bar{V}_{2}$, d'autres facteurs doivent être pris en considération, c'est ainsi que :

331. Le - stress * auquel est soumis le poisson lors de son introduction dans l'enceinte expérimentale est susceptible de modifier considérablement la $\bar{v}_{2}$. Les variations sont particulièrement marquées chez l'animal, non accoutumé aux conditions expérimentales, ou soustrait pendant plusieurs jours à celles-ci (fig 4).

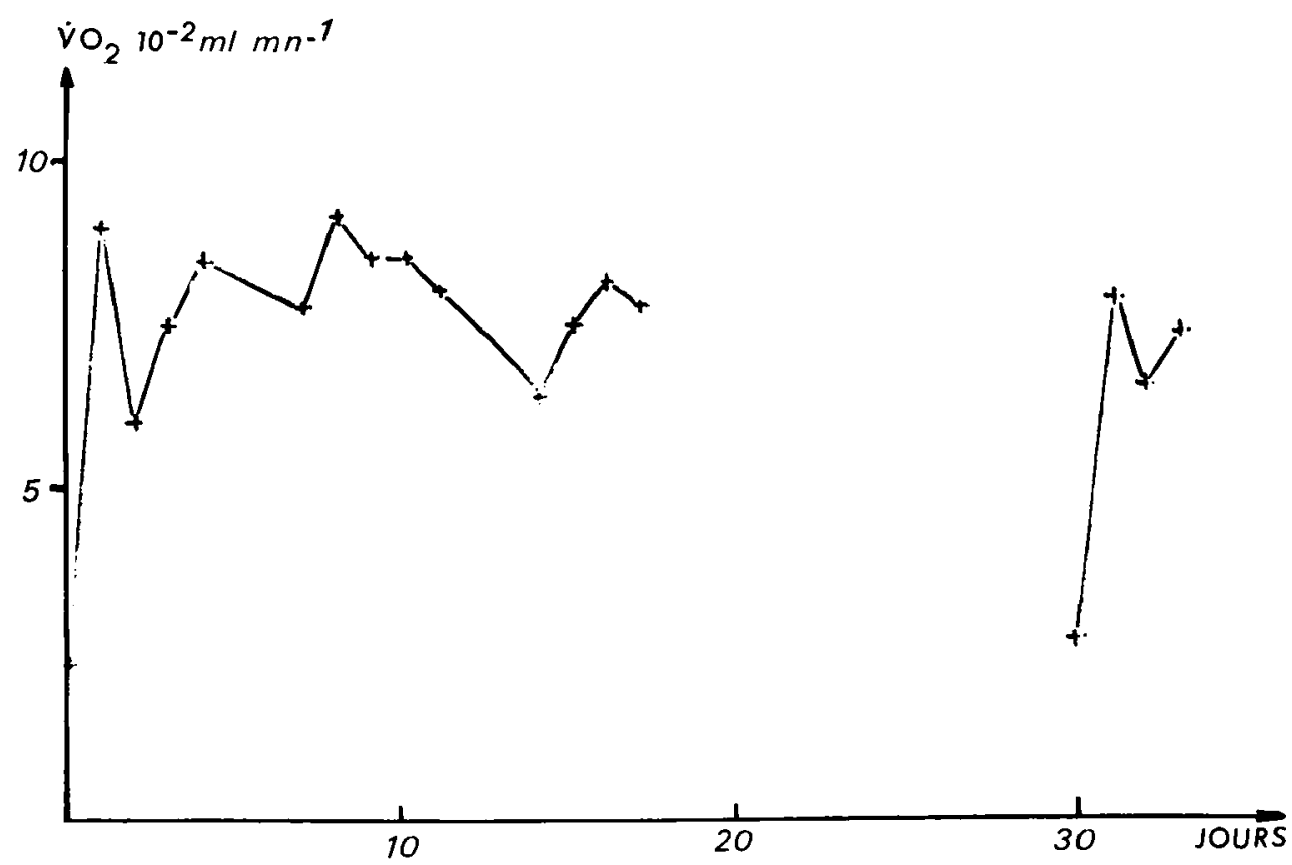

Fig. 4 : Variations journalières de la consommation d'oxygène d'un Gardon dans nos conditions expérimentales 
332. Des stimuli visuels ou vibratoires suffisants augmentent passagèrement la $\overline{\mathrm{V}}_{2}$ (fig 5), alors qu'un stress important ralentit la $\overline{\mathrm{V}} \mathrm{O}_{2}$.

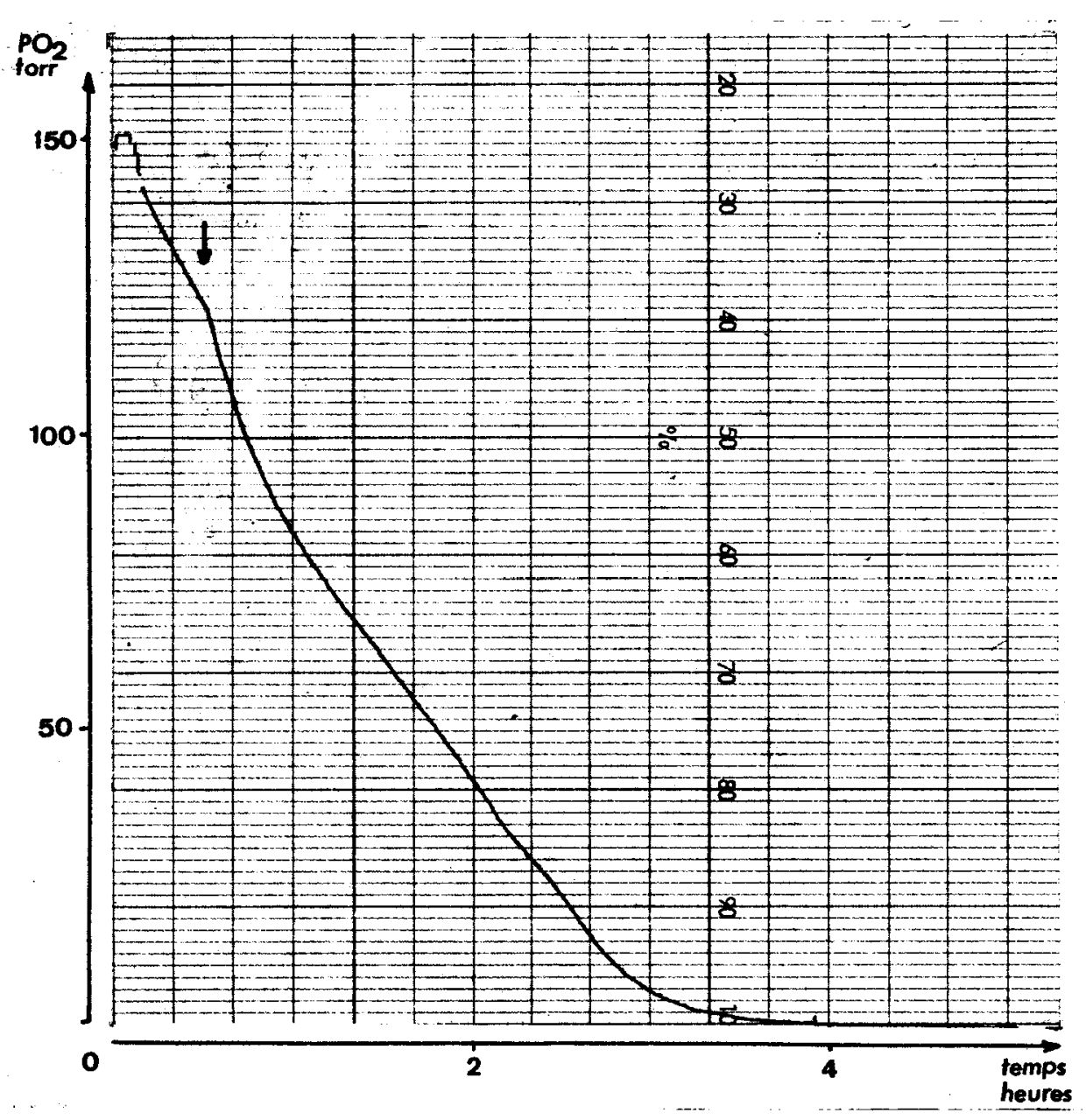

Fig. 5 : Influence d'un stimulus sonore

II semble donc apparaitre une hiérarchie dans les stimuli dont les effets peuvent être inverses.

333. Différents facteurs pathologiques de variations peuvent être mis en évidence. Nous avons observé une variation cyclique (de 18 jours) de la $\overline{\mathrm{O}}_{2}$ chez un Gardon parasite par Ichtyophtirius (fig. 6). Des modifications s'observent en outre peu avant la mort du sujet.

334. De nombreuses substances sont par ailleurs susceptibles de modifier les tracés. Ainsi, en aigü, le benzène à la concentration de 0,6 ppm accélère la $\bar{V}_{2}$, à l'inverse, certains détergents bloquent les échanges respiratoires, probablement par action directe sur les branchies. Le lindane perturbe profondément le métabolisme nerveux et respiratoire et élève en outre le seuil d'hypoxie. 


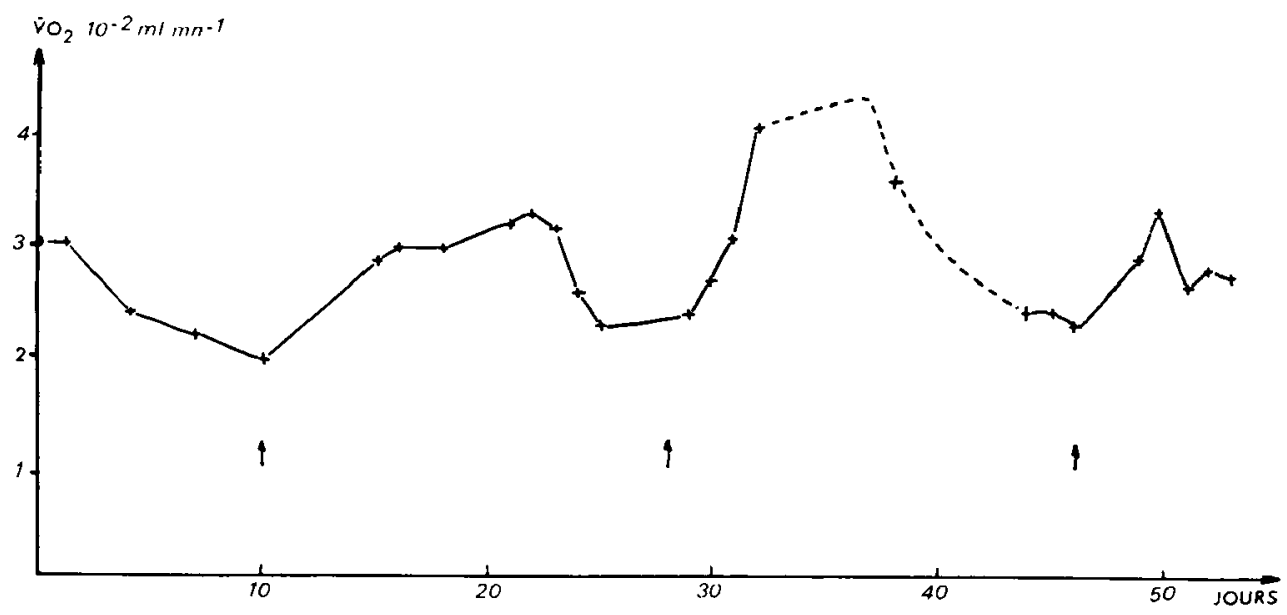

Fig. 6: Graphique de variations cycliques de la consommation d'oxygène chez un Gardon parasité par Ichtyophtirius

335. Enfin, des espèces d'habitats différents se comportent différemment en fonction des conditions hypoxiques du milieu: à $10 \circ \mathrm{C}$ Salmo trutta ne survit pas à 10 torr ; mais Gardonus rutilus supporte $1 \mathrm{~h} 30$ d'anoxie; quant à Ameiurus nebulosus sa résistance à l'anoxie du milieu dépasse 12 heures.

\section{DISCUSSION}

41. Les nombreux facteurs intervenant sur le comportement respiratoire du Gardon, nous ont empêchès de traiter les résultats d'un point de vue statistique.

42. F'ar ailleurs, en l'absence de tout stimulus extérieur provoqué, nous avons observé fréquemment des ruptures de pente dans les tracés témoignant d'une $\overline{\mathrm{V}}_{2}$ accrue, et aucune explication satisfaisante n'a pu être donnée de ce phénomène (modifications endocrines ?).

43. Enfin, si différentes espèces réagissent différemment aux conditions expérimentales, il y a vraisemblablement lieu de tenir compte de l'âge du poisson dans les réponses respiratoires enregistrées.

\section{CONCLUSION}

L'utilisation des électrodes spécifiques à $\mathrm{PO}_{2}$ et $\mathrm{PCO}_{2}$ nous a permis de suivre en continu les échanges gazeux entre un poisson et son milieu environnant au cours du confinement aquatique. Par sa sensibilité, la méthode permet d'avoir à tout moment connaissance de l'activité respiratoire de l'animal, et des modifications de cette activité en fonction de divers facteurs. Cependant, les conditions du confinement empêchent toute mesure de longue durée, aussi avons-nous envisagé de modifier le dispositif, par un circuit extérieur de renouvellement, afin de pouvoir suivre en continu et sur plusieurs jours, voire, plusieurs semaines, le comportement respiratoire du poisson. 
Des travaux préliminaires utilisant ce dispositif modifié nous ont déjà permis des enregistrements de iongue durée, qui ont montré des variations cycliques de l'activité respiratoire chez Salmo Trutta.

Travail effectué avec l'aide d'une subvention du Conseil Scientifique de l'Université de Picardie (Groupe de Recherche Hydrobiologie : Professeur Pierre HARICHAUX).

\section{BIBLIOGRAPHIE}

1. ARRIGNON, J. (1973). * Introduction à l'ètude écologique d'un cours d'eau *. Bul. Fr. de Pisciculture, 251, 71.

2. DEJOURS, P.; ARMAND, J. et VERRIEST, G. (1968) * Carbon dioxide dissociation curves of water and gaz exchange of water breather *. Resp. Physiol., $5,23-33$.

3. HARICHAUX, P. ; POIZOT, A. et FREVILLE, M. (1972). * Mechanism of respiratory exchanges in aquatic environment. A general review including personal results *. R.I.O.M., 27, 71-84.

4. HARICHAUX, P. ; LIBERMANN, I.; GORIN, J. et FREVILLE, M. (1973). - Enregistrement direct en $x y$ du diagramme $\mathrm{PO}_{2}-\mathrm{PCO}_{2}$ dans les conditions du confinement aquatique $n$. J. Physiol., 67, (3), $345 \mathrm{~A}$.

5. HOAR, W.S. ; RANDALL, D.J. * Fish physiology *. Academic Press, NEW-YORK and LONDON.

Vol. I (1969) : Excretion. Ionic regulation and metabolism.

Vol. IV (1970): The nervous system circulation and respiration.

Vol. VI (1971) : Environmental relations and behavior.

6. MAREN, T.H. (1967). "Carbonic anhydrase chemistry, physiology and inhibition . Physiol. Rev., 47, 595-781. 